\title{
A Crystal-based Matter-wave Interferometric Gravitational-wave Observatory
}

\author{
Raymond Y. Chiad* and A. D. Speliotopoulost \\ Department of Physics, University of California, Berkeley, CA 94720-7300
}

(Dated: December 26, 2003)

\begin{abstract}
It is shown that atom interferometry allows for the construction of MIGO, the Matter-wave Interferometric Gravitational-wave Observatory. MIGOs of the same sensitivity as LIGO or LISA are expected to be orders of magnitude smaller than either one. A design for MIGO using crystalline diffraction gratings is introduced, and its sensitivity is calculated.

PACS numbers:
\end{abstract}

*Electronic address: chiao@physics.berkeley.edu URL: physics.berkeley.edu/research/chiao/

$\dagger$ Electronic address: adspelio@uclink.berkeley.edu 


\section{INTRODUCTION}

Roughly speaking, since an atom with mass $m$ "weighs" much more than a photon with frequency $\omega$, all other things being equal, matter-wave interferometers are expected to be more sensitive to inertial effects than light-wave interferometers by a factor of $m c^{2} / \hbar \omega \sim 10^{10}$ if atoms are used [1]. Because of this factor, it is expected that observatories constructed from matter-wave interferometers will be much more sensitive to gravitational waves (GWs) than those constructed with light-based interferometers. This expected increase in sensitivity makes use of the quantum phase of an atomic deBroglie matter wave $\lambda_{d B}=2 \pi \hbar / m v$ where $v<<c$ is the speed of an nonrelativistic object with mass $m$. When a GW passes through an atom interferometer, the speeds of the atoms in the interferometer - and hence their deBroglie wavelengths - will be slightly perturbed. By suitably designing the interferometer, the shifts in the phase of the atoms caused by this perturbation in their deBroglie wavelength will result in a shift in the interference pattern, which can then be measured interferometrically. Like LIGO, this shift can be used to determine the properties of the GW. We shall argue in this paper that using matter-wave interferometry, a Matter-wave Interferometric Gravitational-wave Observatory (MIGO) can be constructed. For the same sensitivity, MIGO is expected to not only be orders of magnitude smaller than laser-based observatories such as LIGO (Laser Interferometer Gravitational-wave Observatory) and LISA (Laser Interferometer Space Antenna), but is also expected to have a frequency response range much broader than current GW observatories.

While classical-based systems such as LIGO and LISA place classical test masses (mirrors) a certain distance way from the central beam splitter of the interferometer, and measures slight shifts in their positions through light-based interferometry, quantum-based systems such as MIGO throw out quantum test masses (atoms) with certain velocities, and measures the quantum phase shift due to slight shifts in their speeds through atom-based interferometry. As we will show below, given a MIGO with a characteristic length $L_{M I G O}$, for the same shot-noise-limited phase shifts as LIGO at $125 \mathrm{~Hz}$ (where LIGO is most sensitive)

$$
L_{M I G O} \approx\left\{B\left(\frac{\hbar \omega_{\gamma}}{m c^{2}}\right) \frac{c}{2 \pi f L_{L I G O}}\right\}^{1 / 2} L_{L I G O},
$$

where $2 B=150$ is the number of times light bounces back and forth in each of LIGO's arms, $\omega_{\gamma}$ is the frequency of the laser used in LIGO, $m$ is the mass of the atom used in 
MIGO, $f$ is the frequency of the GW, and $L_{M I G O}$ is the length of one of LIGO's arms. Thus, $L_{M I G O} \approx 1 \mathrm{~m}$ as compared to $L_{L I G O}=4 \mathrm{~km}$ if a cesium atom is used. Similarly, a comparison, for equal shot-noise-limited phase shift, of a space-based MIGO with LISA leads to a MIGO configuration that at least ten thousand times smaller than LISA.

In this paper, we present one possible configuration for MIGO, and calculate the sensitivity of this configuration to GWs. We also outline the technologies that could potentially be used to construct it. Even though we restrict ourselves to those technologies that have already been demonstrated in various atom diffraction and interferometry experiments, our purpose in this paper is to explore the extreme limits of these technologies, and through these limits, establish the fundamental limitations to MIGO's capabilities. In this vein, we are particularly interested in crystalline diffraction gratings and mirrors.

While the diffraction of helium atoms off of cleaved lithium fluoride crystals was demonstrated by Otto Stern over seven decades ago [2], and single-crystal Bonse-Hart neutron interferometers was used to measure gravitational effects [3] three decades ago, atom interferometers using the natural periodic structure of crystals as reflective diffraction gratings, i. e., crystalline gratings, to make beam splitters, have yet to be demonstrated. We will nevertheless consider only crystalline gratings in this paper. We shall show that MIGO's overall length depends crucially on the periodicity of the diffraction grating used in the beam splitter; the smaller the periodicity, the shorter the interferometer. While in principle any periodic structure can be used to construct diffraction gratings, the smallest of such possible structures is limited by the natural periodicity of crystals. With a periodicity on the order of Ångströms, diffraction gratings made from single crystals will have a periodicity orders of magnitude smaller than current nanofabricated gratings, whereas diffraction gratings based on light have a periodicity in the hundreds of nanometer range. MIGOs constructed with crystalline gratings will thus be the smallest matter-wave-based GW observatories that can conceivably be made.

\section{BRIEF REVIEW OF ATOM INTERFEROMETRY}

Matter-wave interferometry is based on the particle-wave duality principle of quantum mechanics. This principle states that every massive quantum object can have characteristics of both a particle and a wave. Which of these two characteristics it exhibits depends on 
the properties the experimentalist wishes to measure or exploit. By exploiting the wave nature of particles, matter-wave interferometers have been constructed using "elementary" particles such as neutrons [3], as well as "composite" particles such as atoms [4, 5], and $\mathrm{C}_{60}$ fullerene molecules [6].

Like light-wave interferometry, matter-wave interferometers can be divided into three distinctive parts: the source emitting the interfering particle, the "atom optics" consisting of beam-splitters and mirrors, and the detector. Similar to the beams of light in lightwave interferometers, the beams of particles passing through the interferometer picks up a phase shift as well, but now $\Delta \phi=\left(S_{c l}^{\gamma_{1}}-S_{c l}^{\gamma_{2}}\right) / \hbar$, and $S_{c l}^{\gamma_{1}}$ and $S_{c l}^{\gamma_{2}}$ are the action for a particle travelling along the two quasi-classical paths $\gamma_{1}$ and $\gamma_{2}$ along the two arms of the interferometer. Large throughputs of atoms will be needed for MIGO, as well as advanced "atom optics"; we focus therefore here on the atomic beam source, and the beam splitters and mirrors, and refer the reader to Scoles [7] for an review of atomic-beam detectors.

\section{A. Supersonic Sources and 2D Optical Molasses Collimation}

Supersonic sources were first developed in the 1960s by chemists to study of chemical reactions [7], and they have a number of properties superior to the effusive sources (ovens) which are more often used by physicists. While the velocity distribution of atom beams from effusive sources is essentially a Maxwellian one that is fixed by the temperature of the oven, the velocity spreads of supersonic sources are much narrower. Fractional velocity spreads of $1 \%-0.3 \%$ for helium - have been achieved from continuous supersonic sources, and even smaller fractional velocity spreads can be achieved using pulsed sources. Nearly monoenergetic beams with very high intensities can thus be produced. Pritchard [4], for example, has produced $10^{21}$ atoms $/ \mathrm{cm}^{2} / \mathrm{s} / \mathrm{sr}$ sodium beams, and Toennies [8] has produced very cold helium beams with $1.5 \times 10^{19}$ to $1.5 \times 10^{20}$ atom $/ \mathrm{sr} / \mathrm{s}$.

Continuous Supersonic Sources: A typical continuous supersonic source functions as follows: A jet of gas from a high-pressure reservoir escapes supersonically in free expansion through a nozzle, consisting of a small orifice typically 10 to 100 microns in diameter, into a differentially pumped low-pressure chamber that has a larger orifice at its output end called the "skimmer". This skimmer has the appropriate geometry to skim away the hotter components of the outer perimeter of the rapidly expanding gas jet, thus leaving only 
the intense, low-temperature central component of the atomic beam to enter into another differentially pumped chamber. Importantly, after the expansion the atoms in the beam no longer collide with one another. The beam is often collimated yet again using a second slit at the output end of yet another differentially pumped chamber before it enters the main vacuum chamber containing the atom-based device, such as an interferometer 4]. With successive stages of differential pumping by means of either diffusion or turbomolecular pumps, one can maintain an ultra-high vacuum in the main chamber that is often needed. In addition, using optical molasses techniques [9], beam collimation can be achieved without throwing away most of the atomic beam, as is currently done when collimating slits are used. The combination of $2 \mathrm{D}$ optical molasses collimation with effusive sources has been achieved by Kasevich [5]. However, its combination with supersonic sources, as we propose here, can yield the high-brightness atomic beams necessary for good signal-to-noise ratios in MIGO.

Pulsed Supersonic Sources: Most pulsed supersonic sources function in much of the same way as continuous supersonic sources, but with the addition of a fast-acting valve to pulse the beam. Duty cycles of roughly $10^{-4}$ can be achieved resulting in very high fluences. Because of the short duty cycles, smaller pumps than those used in continuous supersonic sources can be used. Of particular interest is the pulse source developed by Powers et. al. 10], which uses laser ablation of metallic sources such as lithium [11] to generate the atomic beam. The high-pressure gas chamber is replaced by the laser-plus-metal assembly, and optical molasses can then be added to reduce the velocity spread of the pulsed supersonic beam as well.

\section{B. Atomic Beam Optics}

There are a number of approaches that can be taken to fabricate the "atom beam optics" - the beam splitters and mirrors - needed for the construction of MIGO. These optics have been constructed by using nanofabricated physical gratings 4, 6], and by also using light through either standing waves or stimulated raman pulses [12]. We shall focus on the possibility of using cleaved surfaces of single crystals, which yield near-perfect crystalline surfaces, in the construction of optical elements for atomic beams.

Atomic beam mirrors can be constructed either out of diffraction gratings, or from specular reflection off of smooth surfaces. Diffraction orders of atomic beams from crystalline surfaces have a history stretching back to Otto Stern's experiments [2], and research in this 
area continues today [13]. Consequently, construction of zero-diffraction order, crystal-based mirrors is certainly possible. Of more interest for MIGO is the work of Holst and Allison, however. They have demonstrated that the flexible, thin, hydrogen-passivated single-crystal silicon wafer surface $\mathrm{Si}(111)-(1 \times 1) \mathrm{H}$ can be used as a specular mirror for focusing helium atomic beams [14]. The development of these mirrors can not only be used to construct collimating optics for the atomic beam, but they also demonstrated that planar, specularreflection atomic mirrors constructed from single crystals are possible as well. We shall see that the sensitivity of MIGO is greater if planar, specular-reflection mirrors are used instead of diffraction gratings as reflection optics, and we will focus primarily on these types of mirrors here.

Since the diffraction of atomic beams off of single crystals has been demonstrated, it is possible, in principle, to construct coherent beam splitters using single-crystal diffraction gratings that work in reflection. Such diffraction gratings have a periodicity of a few Ångströms, and will be orders of magnitude smaller than that of current nanofabricated diffraction gratings. Alignment of interferometers constructed from crystal diffraction grating is much more challenging, however, and it is still an open question as to whether considerations arising from the Debye-Waller factor will allow for the construction of these interferometers. We address both issues below.

Laser Interferometer Alignment: Since crystal-based atom interferometers must be aligned within an Ångström or so, laser interferometry must be used to position and align the various optical elements of these interferometers. Although this is a difficult engineering task, there is no fundamental obstacle towards this goal. The sensitivity of the laser interferometry in distance measurements to make such measurements arises from the photon-shot noise limit $\delta \varphi_{\text {laser }} \simeq 1 /\left\{\dot{N}_{\text {photon }} \tau\right\}^{1 / 2} \simeq 1 \AA / 1 \mu \mathrm{m} \simeq 10^{-4}$, where $\dot{N}_{\text {photon }} \tau$ is the number of photons in a given measurement time $\tau$ interval. Hence we would need $\dot{N}_{\text {photon }} \tau \simeq 10^{8}$ in order to perform these measurements. This is not difficult achieve with standard lasers.

The argument in support of the feasibility of aligning crystal-based atom interferometers is further strengthened when one realizes what has already been achieved by LIGO. Unlike LIGO, where the mirrors of the interferometer are in free fall, i.e., placed as pendula on the end of kilometer-long interferometer arms, as we shall see below, the mirrors and beam splitters in MIGO can be fixed to an underlying, mechanically rigid frame. Furthermore, 
the size of MIGO is on the order of meters, and not of kilometers as in the case of LIGO, or of millions of kilometers as in the case of LISA. This should make the task of aligning MIGO orders of magnitude easier than that for LIGO. Indeed, the active-feedback-mechanism technology developed for LIGO to stabilize the mirror-assembly-central-beam-splitter distance to within $10^{-13} \mathrm{~m}$ could be transferred to MIGO as well [15].

Decoherence and the Debye-Waller factor: Quantum interference requires that there exists no possibility of "which-path" information for the helium atom inside the interferometer. This requires that diffraction of atomic beams off of beam splitters, and their reflection off of mirrors be elastic and coherent. (Scattering of atoms in the beam off of background gas in the ultra-high vacuum system is assumed to be negligible). A measure of the inelastic versus elastic components of these processes is based on the Debye-Waller factor $W$ in the intensity ratio $I / I_{0}=\exp (-2 W)$, where $I$ is the diffracted (or reflected) intensity and $I_{0}$ is the incident intensity. The factor $W$ is a measure of the fluctuations of the atoms in the crystal that diffract the incident helium beam. For diffracted helium beams, $W=\mathcal{B} / a^{2}$, where $a$ is the lattice constant of the crystal, and $\mathcal{B} \sim 0.5 \AA^{2}$ at room temperature [16]. The rule of thumb [7] is that $W / 12<0.1$, for sharp, elastic diffraction patterns to be seen. The requirement for observing interference is more stringent, however; the probability of emitting even a single phonon during the diffraction process must be negligible, and $W / 12<0.01$ is

required [17]. For crystal diffraction grating and mirrors, $W / 12 \sim 10^{-3}$ at room temperature and decreases at lower temperatures. Thus it is highly probable that the zero-phonon process is the dominant one, and therefore quantum phase coherence is expected in the interferometer. The observation of high-visibility interference fringes for neutrons in the Bonse-Hart interferometer [3] is evidence that this is indeed the case.

\section{LIGO, LISA, AND THE DETECTION OF GWS}

The great majority of the current experimental searches for GWs are based on laser interferometry. These detectors are scalable by design to such sizes that the detection of GWs becomes feasible. A number of research groups located throughout the world [18] are expecting to begin to collect data soon: GEO600, German-British collaboration; VIRGO, a French-Italian collaboration; TAMA300, a Japanese effort; and ACIGA, an Australian effort. The current US-based, international collaboration, is LIGO. In addition, a space- 
based laser interferometer system LISA is currently in the initial planning stage. We will focus specifically on LIGO.

LIGO is a set of three interferometers based at two locations separated by $3020 \mathrm{~km}$ : Hanford, Washington and Livingston, Louisiana [15]. All three instruments are based on Michelson interferometers with Fabry-Perot arms. The physical length of the each arm of the main LIGO interferometer is $4 \mathrm{~km}$, and with the Fabry-Perot interferometers in each arm, the optical path of the arm is increased by the bounce factor, $2 B=150$. At the end of each arm is a massive mirror suspended vertically as pendula within a vacuum chamber, and the location of the mirror assembly must be held in position within $10^{-10}$ to $10^{-13} \mathrm{~m}$ with respect to the center of the interferometer.

In its current LIGO I configuration, LIGO is designed to detect GWs in the frequency range of 40 to $10^{4} \mathrm{~Hz}$. At frequencies below $40 \mathrm{~Hz}$ seismic noise causes a rapid decrease in LIGO's sensitivity, and at frequencies above $125 \mathrm{~Hz}$ the shot-noise limit ${ }^{14}$ begins to gradually limit LIGO's sensitivity as well. Between 40 and $125 \mathrm{~Hz}$ LIGO's sensitivity is limited by thermal noise in the suspension system of the end mirrors. Construction of LIGO I began in 1996, and the main interferometers were commissioned in 2001. The first science runs were started in June of 2002; data from these runs are currently being analyzed. Upgrading LIGO I to LIGO II, which is designed for GW astronomy, is expected to begin in 2006 and to be completed by 2007 .

\section{THE $M$ ATTER-WAVE INTERFEROMETRIC GRAVITATIONAL-WAVE OBSERVATORY (MIGO)}

\section{A. The Vertical MIGO Configuration}

Fig. 1 is a diagram of the vertical MIGO configuration, one of the possible configurations for MIGO. We start here with a simplified description of the interferometer. Detailed analysis of the response of this configuration to the passage of GWs will be presented below.

In the vertical configuration for MIGO shown in Fig. 1, alkali atoms are emitted from a pulsed supersonic source, and are then slowed down using Zeeman slower 9] to velocities so slow that the acceleration due to gravity $g$ dominates the trajectories of the atoms through the interferometer. Immediately after the supersonic source, the beam is then collimated 
using 2D optical molasses to further narrow the transverse velocity spread of the atomic beam. After the beam is diffracted off the initial beam splitter atoms in the $n= \pm 1$ orders, mirrors, which retroreflect the almost parabolic upwards trajectories, are placed at the top of these trajectories. (Atoms in the higher diffraction orders are not used.) The atoms are then reflected off of these mirrors and fall back downwards, almost retracing their almost-parabolic upwards trajectories. The left and right paths of the falling atoms are then recombined by the same beam splitter, and subsequently detected. The bilateral symmetry of this interferometer along the vertical line ensures that the gross motion of the atoms on the left side of the interferometer is reflected on the right side. The parabolic trajectories of the atoms is shifted slightly during the passage of a GW, and, as can be seen from the force lines in Fig. 1, will be slightly different for atoms travelling along the left hand path compared to the right hand path. It is this asymmetrical shift of the atomic trajectories by a $\times$-polarized GW that leads to the overall phase shift.

\section{B. MIGO and LIGO: The Underlying Physics}

In this section, we focus on the physics that underlies both MIGO and LIGO, and compare their relative sensitivities to GWs from astrophysical sources. We shall emphasize only the physics here, and our arguments will be more physical than formal; an exact calculation of the MIGO phase shift can be found in the next section. As such, in this section we consider only the effects of a GW passing over freely-falling particles, and neglect all other external forces on the system.

As is well known, when a GW passes through a system, its effects on all parts of the system [19, 20] must be included. It is not possible to shield against gravitational forces as it is for the other forces of nature. Only differences in the motion between objects - such as the mirrors and the beam splitters in an interferometer-can be measured; their absolute motion cannot. As a consequence, the motion of an object relative to an observer is governed by the geodesic deviation equation [19] containing an effective tidal acceleration acting on the object arising from the local curvature of the spacetime. In the long wavelength limit, this equation reduces to

$$
\frac{d^{2} x^{i}}{d t^{2}}=\frac{1}{2} \frac{d^{2} h_{i j}}{d t^{2}} x^{j}
$$

where by construction the origin of coordinate system is placed at the observer at the beam 
splitter. The metric deviations from flat spacetime $h_{i j}(t)$, i. e., the amplitude of the GW, is a $3 \times 3$ tensor, and not a vector as it is for electromagnetic waves, although GWs have two polarizations - the + polarization and the $\times$ polarization-just like electromagnetic waves.

The geodesic deviation equation lies at the heart of both LIGO and MIGO: LIGO on the classical level, and MIGO on the quantum level. For LIGO, one considers a test mass (a mirror) at a distance $L^{i}$ from the observer (the central beam splitter), which is taken to be the origin of the coordinate system. When a GW passes over the apparatus, it causes small shifts $\xi^{i}$ in this distance so that since $x^{i}=L^{i}+\xi^{i}$, where $\left|\xi^{i}\right|<<\left|L^{i}\right|$,

$$
\frac{d^{2} \xi_{i}}{d t^{2}} \approx \frac{1}{2} \frac{d^{2} h_{i j}}{d t^{2}} L^{j}
$$

To lowest order,

$$
\xi_{i} \approx \frac{1}{2} L^{j} \int_{0}^{t} d t^{\prime} \int_{0}^{t^{\prime}} d t^{\prime \prime} \frac{d^{2} h_{i j}}{d t^{2}}=\frac{1}{2} L^{j} h_{i j}
$$

where for $t \leq 0$, we assume $h_{i j} \equiv 0$. Thus, in LIGO one puts a mass (a mirror) at $t=0$ a certain distance $L^{i}$ away from the observer, and observes fluctuations in its position due to the passage of a GW. These fluctuations are measured by light-based interferometry. The freely-falling end mirrors of LIGO are designed to be these classical test masses, and the phase shift due to changes in the length of its arms is $\Delta \phi_{L I G O}=2 \pi \Delta L_{L I G O} / \lambda_{\gamma}$, where $\lambda_{\gamma}$ is the wavelength of the laser, and $\Delta L_{L I G O}$ is the optical path length difference of LIGO induced by the GW. For the two arms of the interferometer this difference for a + polarization $\mathrm{GW}$ with amplitude $h_{+}$is $\Delta L_{L I G O}=B L_{L I G O} h_{+}$where the factor of $B$ accounts for multiple reflections of the light beam within each arm of LIGO within the Fabry-Perot interferometers 22]. Thus,

$$
\Delta \phi_{L I G O}=\frac{2 \pi}{\lambda_{\gamma}} B L_{L I G O} h_{+},
$$

and depends only on the amplitude of the GW, and the length $L_{L I G O}$ of LIGO's arms. Strictly speaking this expression for $\Delta \phi_{L I G O}$ is only applicable for GWs with frequencies $\sim 125 \mathrm{~Hz}$; at $4 \mathrm{~km}$, the arms of LIGO is roughly 1/6 the wavelength of a GW in the upper end of its frequency response spectrum, and causality has to be taken into account [21]. Although design characteristics of LIGO such as power recycling and shot-noise modifications by the Fabry Perot arms will change the sensitivity from this simple form, it is adequate for our purposes. Note that in LIGO the photons used in making the measurement are not the test particles being acted on by the GW; only the end mirrors of the interferometer are being acted on. 


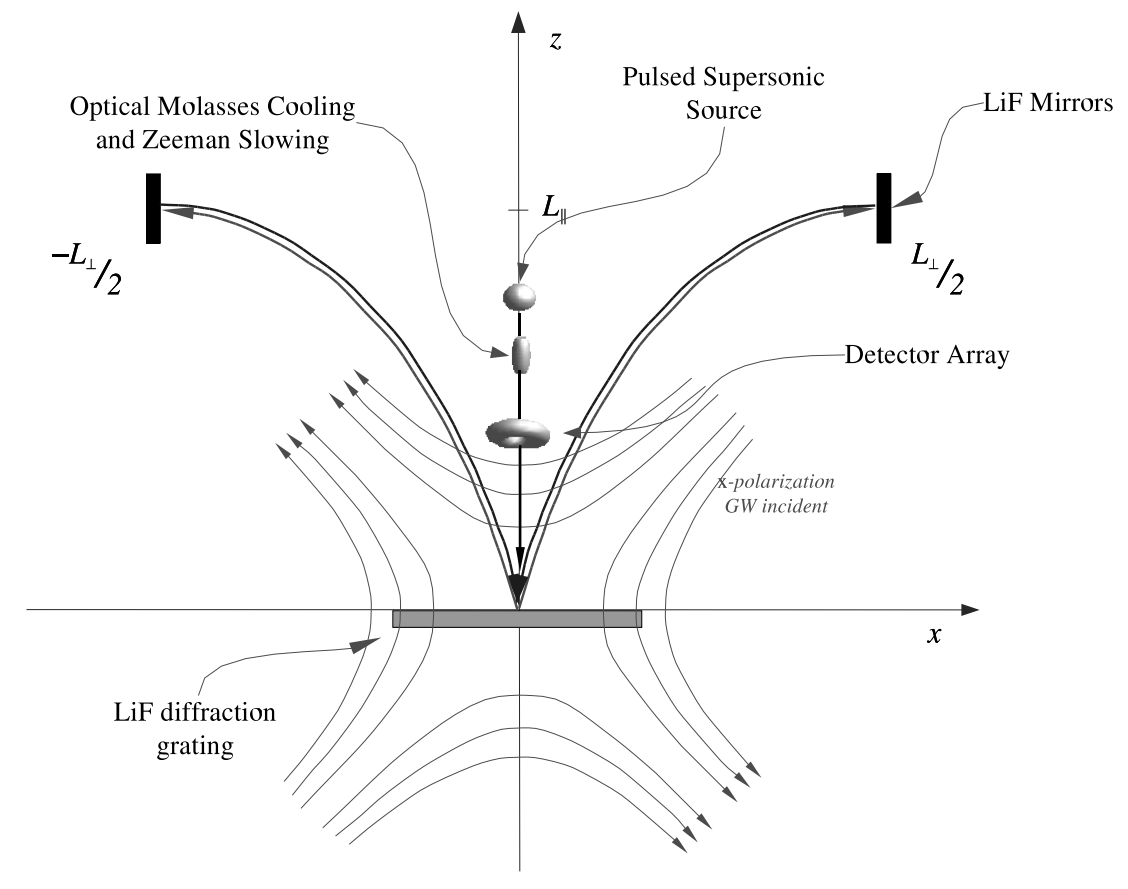

FIG. 1: Schematic of vertical MIGO configuration, with a $\times$ polarization GW incident normal to the plane of the interferometer.

In contrast with LIGO which places an end mirror at a certain distance away and measures shifts in its position, in MIGO one throws out a test mass, i. e., an atom, at $t=0$ with a velocity $V_{i}$ from the origin, and observes deviations in its velocity, and thus in its path in spacetime, due to the passage of a GW. To see this, we take $v_{i}=V_{i}+\beta_{i}$, where $\left|\beta_{i}\right|<<\left|V_{i}\right|$. Then from Eq. (2),

$$
\frac{d \beta_{i}}{d t} \approx \frac{1}{2} \frac{d^{2} h_{i j}}{d t^{2}} V^{j} t
$$

Once again to lowest order,

$$
\beta_{i} \approx \frac{1}{2} V^{j} \int_{0}^{t} t^{\prime} d t^{\prime} \frac{d^{2} h_{i j}}{d t^{2}} \sim \frac{1}{2} V^{j} t \dot{h}_{i j}
$$

where $\dot{h}_{i j}$ is the rate of change of the strain field $h_{i j}^{G W}$ of the GW. The parallelism between the displacement $\xi^{i}$ in LIGO, and the velocity shift $\beta_{i}$ in MIGO, is readily apparent. Classically, the velocity shift $\beta_{i}$ is extremely small, and virtually impossible to measure. Quantum mechanically, however, this velocity shift can be measured by means of quantum interference.

A quantum particle such as an atom in a matter-wave interferometer has a deBroglie 
wavelength $\lambda_{d B}=2 \pi \hbar / m v$, where $m$ is the mass of the atom and $v$ is its speed. Changes in the atom's speed $\Delta v$ due to GWs result in changes to its local deBroglie wavelength, and hence its quantum phase. If $T$ is the time it takes the atom to traverse MIGO, and if $L_{M I G O}$ is the characteristic size of MIGO, then $\left|V_{i}\right| \sim L_{M I G O} / T$, and the shift in the atoms position $\Delta L_{M I G O}$ after it traverses the interferometer is $\Delta L_{M I G O} \sim L_{M I G O} T \dot{h}_{\times} / 2$ for a $\times$ polarization GW with amplitude $h_{\times}$. Like LIGO, this results in a phase shift, but now of the form

$$
\Delta \phi_{M I G O} \sim \frac{4 \pi}{\lambda_{\perp d B}} \Delta L_{M I G O}=2 \pi \frac{m}{\hbar} L_{M I G O}^{2} f h_{\times}
$$

where $f$ is the frequency of the $\mathrm{GW}$, and the deBroglie wavelength of the atom is now used instead of the wavelength of light. Thus, in contrast to LIGO, MIGO's phase shift $\Delta \phi_{M I G O}$ is proportional to the rate of change of the GW amplitude. Although simplistic, this rough derivation of the MIGO phase shift nevertheless elucidates the underlying physics of MIGO. The above expression for the MIGO phase shift is close to the exact equation we shall derive in the next section. Equation (14) follows directly from Eqs. (3) and (4) by setting $\Delta \phi_{M I G O}=\Delta \phi_{L I G O}$, and the amplitudes of the two polarizations of the GW equal.

The relative sizes of MIGO compared with LIGO given in Eq. (1I) would seem to be counterintuitive. MIGO makes use of slowly-moving, nonrelativistic atoms to make its measurements, while LIGO would seem to make use of fast-moving photons. At first glance it would seem that MIGO should be less sensitive to GWs than LIGO by some power of $v_{\text {atom }} / c$. This, however, would be an erroneous argument. As outlined in the above, it is not the effect of the $\mathrm{GW}$ on the photons used in the laser interferometer that is being measured in LIGO; it is the effect of the GW on the test masses (the mirrors) which are at rest that is being measured.

\section{Phase Shift Calculation}

In this section we present an explicit calculation of the vertical MIGO phase shift. As shown in Fig. 1 the origin of our coordinate system is place at the base of the beam splitter. In the absence of a $\mathrm{GW}$ the atoms travel along parabolic trajectories. If a $\mathrm{GW}$ with $h_{i j}(t)$ is incident along the $y$-axis, these parabolic paths will be slightly perturbed, and we can calculate the resulting phase shifts for the atoms by taking $\vec{x}(t)=\vec{x}_{0}(t)+\vec{x}_{1}(t)$ where $\vec{x}_{0}(t)$ is the path of the atoms in the absence of a GW, and $\vec{x}_{1}(t)$ is the first-order perturbation 
to this path.

If the $r$ th atom is sent through the beam splitter at $t=t_{r}$, the path of the atom in the $n=+1$ diffraction order in the absence of a GW is: $v_{0 x}=v_{\perp}, v_{0 z}=v_{\|}-g\left(t-t_{r}\right)$, $x_{0}=v_{\perp}\left(t-t_{r}\right)$, and $z_{0}=v_{\|}\left(t-t_{r}\right)-g\left(t-t_{r}\right)^{2} / 2$ for $t_{r}<t<t_{r}+T / 2$, where $T / 2$ is the time for the atom to travel from the beam splitter to the mirror, and $g$ is the local acceleration due to Earth's gravity. Since our focus is on GWs, we approximate $g$ as a constant, and neglect local curvature effects from stationary sources such as the Earth. For $t_{r}+T / 2<t<t_{r}+T$. during which the atom travels from the mirror back to the beam splitter, its path is $v_{0 x}=-v_{\perp}$, $v_{0 z}=v_{\|}-g\left(t-t_{r}\right), x_{0}=L_{\perp}-v_{\perp}\left(t-t_{r}\right)$, and $z_{0}=v_{\|}\left(t-t_{r}\right)-g\left(t-t_{r}\right)^{2} / 2$, where $L_{\perp}=v_{\perp} T$ is the width of the interferometer and $L_{\|}=g L_{\perp}^{2} / 8 v_{\perp}^{2}$ is its height. The velocities $v_{\perp}$ and $v_{\|}$are the initial velocities of the atom as it leaves the diffraction-grating beam splitter, and $v_{\perp}=2 \pi \hbar / m a$ where $a$ is the periodicity of the grating; $v_{\|}=\left(v_{s}^{2}-v_{\perp}^{2}\right)^{1 / 2}$ where $v_{s}$ is the velocity of the beam incident the beam splitter. A similar set of equations hold for the $n=-1$ order with $v_{\perp} \rightarrow-v_{\perp}$.

The perturbed path $\vec{x}_{1}$ satisfies the geodesic deviation equations of motion in Eq. (2), but with $\vec{x}$ approximated as $\vec{x}_{0}$ on the right hand side. Like the unperturbed system, we solve the equations of motion for $\vec{x}_{1}$ by dividing the trajectory of the atom into two parts: first from the beam splitter to then mirrors, and then from the mirrors back to the beam splitter. The effect of the mirrors on the paths of the atoms are taken into account through the mirror boundary conditions $v_{1 z}\left(t_{r}+T^{-} / 2\right)=v_{1 z}\left(t_{r}+T^{+} / 2\right)$ and $v_{1 x}\left(t_{r}+T^{-} / 2\right)=-v_{1 x}\left(t_{r}+T^{+} / 2\right)$; the mirrors are taken to be rigidly mounted on the frame of the interferometer. The - and + superscripts denote the atom approaching and leaving the mirror, respectively. This jump boundary condition on the velocity of the atoms along the $x$-axis is due to the instantaneous, impulsive force the mirror exerts on the atom when it hits the mirror.

The action for the geodesic deviation equation can be written as

$$
S=m \int d t\left(\frac{1}{2} v_{i} v^{i}-\frac{1}{2} \dot{h}_{i j} x^{i} v^{j}-g z\right)
$$

Writing $v^{i}=v_{0}^{i}+v_{1}^{i}$, we find that for the $n=+1$ path the change in the atom's action $S_{+1}^{\prime}$ 
due to the GW relative to the unperturbed action is [20]

$$
\begin{aligned}
\frac{S_{+1}^{\prime}\left(t_{r}\right)}{m} \approx & \frac{1}{2} L_{\perp}\left[v_{1 x}\left(t_{r}+T^{-}\right)-v_{1 x}\left(t_{r}+T^{+}\right)\right]+\frac{1}{2} L_{\perp} v_{\perp} h_{x x}\left(t_{r}+T / 2\right) \\
& +L_{\|} v_{\perp} h_{x z}\left(t_{r}+T / 2\right)-\frac{1}{2} \int_{t_{r}}^{t_{r}+T} d t\left\{h_{x x}(t) v_{\perp}^{2}+h_{z z}(t) v_{0 z}^{2}(t)\right\} \\
& -v_{\perp}\left\{\int_{t_{r}}^{t_{r}+T / 2} d t h_{x z}(t) v_{0 z}(t)-\int_{t_{r}+T / 2}^{t_{r}+T} d t h_{x z}(t) v_{0 z}(t)\right\} \\
& +\frac{1}{2} g \int_{t_{r}}^{t_{r}+T} d t\left[h_{x z}(t) x_{0}(t)+h_{z z}(t) z_{0}(t)-2 z_{1}(t)\right],
\end{aligned}
$$

after successive integration by parts, and using the geodesic deviation equation of motion. For the $n=-1$ path, we get $S_{-1}^{\prime}\left(t_{r}\right)$ from $S_{+1}^{\prime}\left(t_{r}\right)$ by taking $v_{\perp} \rightarrow-v_{\perp}$ in the above. Then,

$$
\begin{aligned}
\frac{\hbar}{m} \Delta \phi_{M I G O} \approx & L_{\perp}\left[v_{1 x}^{+1}\left(t_{r}+T^{-} / 2\right)+v_{1 x}^{-1}\left(t_{r}+T^{-} / 2\right)\right]+ \\
& 2 v_{\perp} L_{\|} h_{x z}\left(t_{r}+T / 2\right)-2 v_{\perp}\left\{\int_{t_{r}}^{t_{r}+T / 2} d t h_{x z}(t) v_{0 z}(t)\right. \\
& \left.-\int_{t_{r}+T / 2}^{t_{r}+T} d t h_{x z}(t) v_{0 z}(t)\right\}+g \int_{t_{r}}^{t_{r}+T} d t h_{x z} x_{0}(t) \\
& -g \int_{t_{r}}^{t_{r}+T} d t\left[z_{1}^{+1}(t)-z_{1}^{-1}(t)\right]
\end{aligned}
$$

where the superscripts \pm 1 denote the trajectories for the \pm 1 orders, and we have used the fact that $x_{0}^{+1}(t)=-x_{0}^{-1}(t)$, while $z_{0}^{+1}(t)=z_{0}^{-1}(t)$. Then,

$$
\left(v_{1 x}^{+1}+v_{1 x}^{-1}\right)\left(t_{r}+T^{-} / 2\right)=L_{\|} \dot{h}_{x z}\left(t_{r}+T / 2\right)+v_{\|} h_{x z}\left(t_{r}\right)-g \int_{t_{r}}^{t_{r}+T / 2} d t h_{x z}(t),
$$

from Eq. (2), while for $t_{r}<t<t_{r}+T / 2$,

$$
\left(z_{1}^{+1}-z_{1}^{-1}\right)(t)=x_{0}(t)\left[h_{x z}(t)+h_{x z}\left(t_{r}\right)\right]-2 v_{\perp} \int_{t_{r}}^{t} d t^{\prime} h_{x z}\left(t^{\prime}\right),
$$

and for $t_{r}+T / 2<t<t_{r}+T$,

$$
\begin{aligned}
\left(z_{1}^{+1}-z_{1}^{-1}\right)(t)= & L_{\perp} h_{x z}(t)-v_{\perp}\left[t-t_{r}\right]\left\{h_{x z}(t)-h_{x z}\left(t_{r}\right)\right\} \\
& -2 v_{\perp} h_{x z}\left(t_{r}+T / 2\right)\left[t-t_{r}-T / 2\right] \\
& +2 v_{\perp}\left(\int_{t_{r}+T / 2}^{t} d t^{\prime} h_{x z}\left(t^{\prime}\right)-\int_{t_{r}}^{t_{r}+T / 2} d t^{\prime} h_{x z}\left(t^{\prime}\right)\right) .
\end{aligned}
$$

Integrating, and then taking the Fourier transform, we get

$$
\Delta \phi_{M I G O}(f)=-2 \pi \frac{m}{\hbar} L_{\perp} L_{\|} i f h_{\times}(f) e^{-i \pi f T} F_{v}(f T / 4),
$$


for a GW with amplitude $h_{\times}(f)$ and frequency $f$. Only the $\times$ polarization causes a net phase shift in the interferometer; the + polarization does not, due to the bilateral symmetry of the interferometer. The resonance factor is

$$
F_{v}(f T / 4)=1-\{\operatorname{sinc}(\pi f T / 2)\}^{2}+\frac{i}{\pi f T / 2}\{1-\operatorname{sinc}(\pi f T)\}
$$

where $\operatorname{sinc}(x) \equiv \sin x / x$. Note that $F_{v} \rightarrow 1$ as $f T \rightarrow \infty$, and thus MIGO is most sensitive to GW in the high frequency domain. This sensitivity is a direct result of the specular reflection mirrors, and their effects on the atoms are embodied by the jump boundary conditions that we used. If diffraction-grating mirrors were used instead, the high-frequency sensitivity of MIGO would be drastically reduced.

While $\Delta \phi_{M I G O} \propto L_{\perp} L_{\|}$, because the acceleration due to gravity, $L_{\|}=g L_{\perp}^{2} / 8 v_{\perp}^{2}$, where $v_{\perp}$, the horizontal velocity of atom after the beam splitter, is inversely proportional to the periodicity of the diffraction grating used. In particular, we see that $L_{\|} \propto a^{2}$; the smaller the periodicity of the diffraction grating, the shorter the interferometer; hence the use of crystalline diffraction gratings. We thus see $\Delta \phi_{M I G O}$ for the vertical MIGO configuration is proportional to $L_{\perp}^{3}$, and not to $L_{\perp}$ as it is for LIGO. Indeed, taking the absolute value of $\Delta \phi_{M I G O}$, the shot-noise-limited sensitivity for the vertical MIGO is

$$
\tilde{h}(f)_{\text {shot }}^{M I G O}=\frac{\hbar}{2 \pi m L_{\perp} L_{\|} f\left|F_{v}(f T / 4)\right| \dot{N}^{1 / 2}}
$$

where $\dot{N}$ is the number of atoms passing though the interferometer per second. In comparison, $\tilde{h}(f)_{\text {shot }}^{L I G O}=\left\{\hbar \omega_{\gamma} / 2 I_{o} \eta c\right\}^{1 / 2}\left(2 \pi f / \omega_{\gamma}\right)$, where $I_{o}$ is the power of the laser, and $\eta$ is the

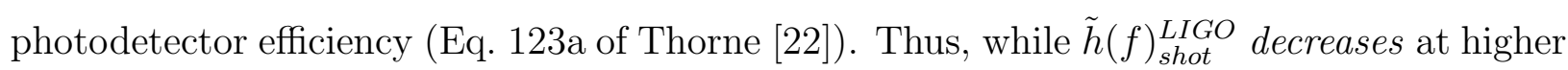

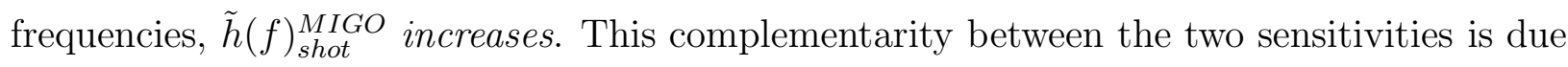
to fundamental differences in the signals measured by LIGO and MIGO: LIGO measures position, while MIGO measures velocity.

In the above analysis the mirrors of the interferometer is assumed to be rigidly attached an infinitely-rigid interferometer that does not move when the GW passes through. This is an approximation. A more accurate model is to attach each mirror to a spring with a resonance frequency $f_{0}$, which depends on the size of the interferometer as well as its material properties, and a quality factor $Q$. When the GW passes through the interferometer it will induce a velocity shift in the mirror, which will in turn induce an additional shift in the atom's velocity. The net affect is to subtract the term $-f^{2} /\left(f^{2}-f_{0}^{2}+i f_{0} f / Q\right) / 2$ from $F_{v}$. 


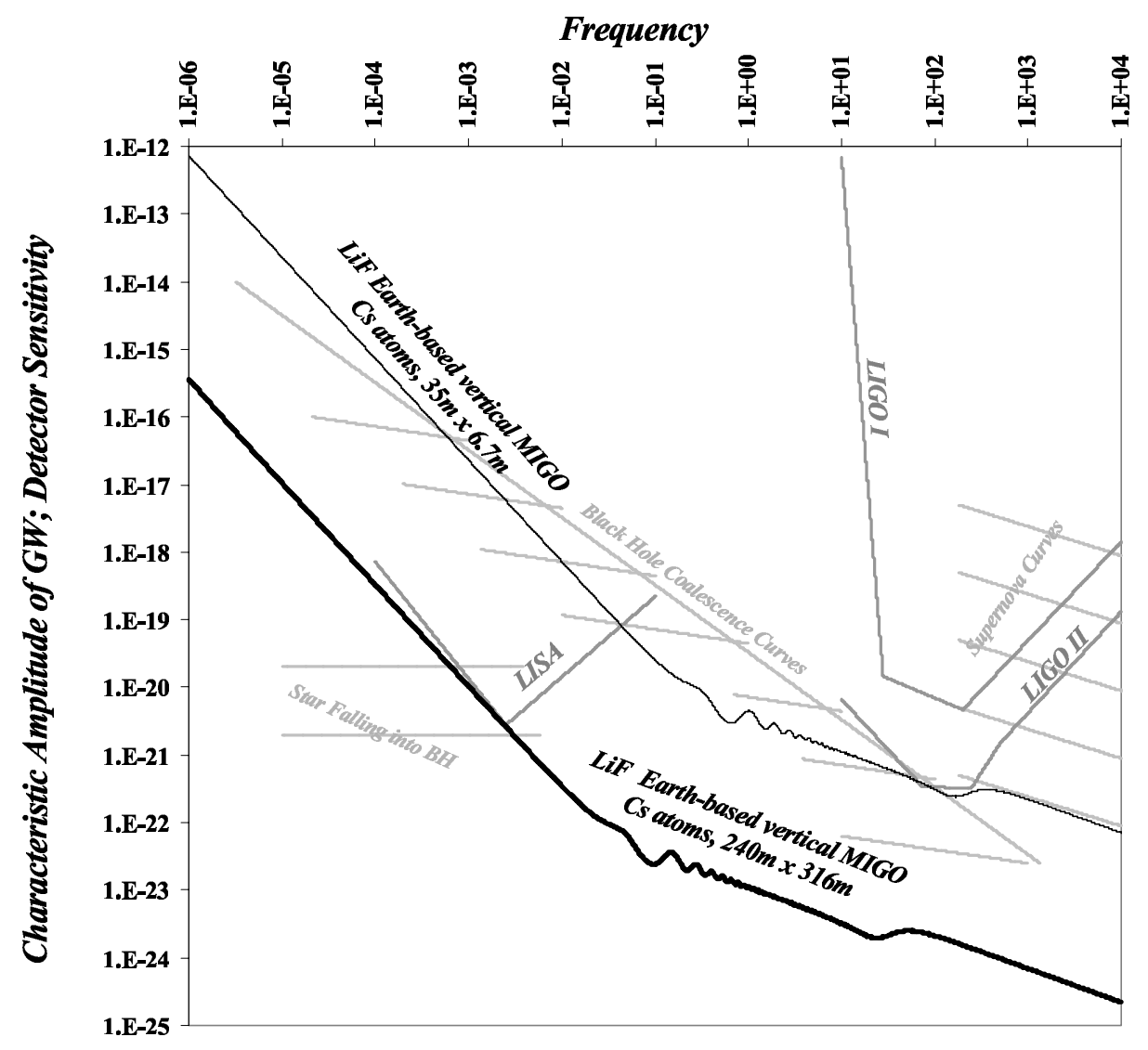

FIG. 2: The ability of MIGO to detect GWs from various classes of burst sources is plotted, and compared with that of LIGO I, LIGO II, and LISA.

The small dips at the high-frequency end of the MIGO graphs shown in Fig. 2 are due to the shift in the mirror's position by the GW where a speed of sound of $6000 \mathrm{~m} / \mathrm{s}$ was taken for the material used in constructing MIGO, and the mounting of the mirror to the frame has a $Q=1$.

\section{Potential Sensitivity}

The graphs for MIGO are plotted using $\tilde{h}_{M I G O}$, and $\dot{N}=10^{18}$ atoms per second. An atomic beam of ${ }^{133} \mathrm{Cs}$ atoms from a $2000 \mathrm{~K}$ pulsed supersonic source is slowed down using a Zeeman slower, and additional 2D optical cooling is used to narrow the velocity spread of the atoms perpendicular to the beam. A LiF crystal, which has a lattice constant of $2 \AA$, is 
used reflection diffraction grating for the beam splitter; the mirrors are constructed out of single LiF crystals as well. Two sizes of MIGOs are graphed. The first has a width $L_{\perp}=35$ $\mathrm{m}$ and a height $L_{\|}=6.7 \mathrm{~m}$; the atoms in the beam of this MIGO must be slowed by a factor of 69 by the Zeeman slower. The second has a width $L_{\perp}=240 \mathrm{~m}$ and a height $L_{\|}=316 \mathrm{~m}$; the atoms in the beam must be slowed by a factor of 10 by the Zeeman slower in this case. Graphs of GW signals from burst sources in the figure were replotted from Thorne's [22] Fig. 9.4. The plots LIGO I, LIGO II and LISA have been updated from Thorne's original figure, and were generated from Thorne [22], Fritschel, and Cutler [23], respectively, using Eq. 111 of Thorne [22].

At $35 \mathrm{~m} \times 6.7 \mathrm{~m}$, the smaller of the two MIGO configurations graphed has the same sensitivity as LIGO II, in LIGO II's operating regime, while being a very small fraction of its size. Similarly, at $250 \mathrm{~m} \times 316 \mathrm{~km}$, the larger of the two MIGO configurations has the same sensitivity as LISA in LISA's operating regime, even though its length is only one tenthousandths of LISA's length of 5 million km. Importantly, in contrast to LISA, this MIGO is earth-based rather than space-based. Both MIGO configurations are sensitive to a wider range of GW frequencies than LIGO and LISA, and they would extend the reach of GW observatories into frequency ranges - in particular between 0.1 and $10 \mathrm{~Hz}$ - not currently accessible by either LIGO or LISA. Note also the sensitivity to high-frequency GWs of all three MIGO configurations graphed in Fig. 3, compared with the gradual loss of sensitivity of LIGO at frequencies above $125 \mathrm{~Hz}$, and of LISA at frequencies above $2 \times 10^{-2} \mathrm{~Hz}$. This is due to differences in the signal being measured by matter-wave- and by light-wave-based interferometers: MIGO measures shifts in the atom's velocity, and thus the rate of change of the GW amplitude with time; the larger the velocity shift, the larger the phase shift. This rate of change increases at higher frequencies, and is the underlying reason why the smallest-amplitude GW that the three MIGO configurations can detect in Fig. 3 decreases as $1 / f^{1 / 2}$ at high frequencies. In contrast, LIGO (and LISA) measures the position of the end mirrors, and depends only on the amplitude of the GW. Indeed, because a GW is time varying, the smallest-amplitude GW that LIGO can detect must increase as $f^{1 / 2}$; it actually increases as $f^{3 / 2}$ because of shot-noise limits to $\Delta \phi_{L I G O}$. This difference between MIGO and LIGO can as be explicitly seen by comparing

$$
\frac{\tilde{h}(f)_{s h o t}^{M I G O}}{\tilde{h}(f)_{s h o t}^{L I G O}} \approx \frac{\hbar \omega_{\gamma}}{m c^{2}}\left(\frac{2 \dot{N}_{\gamma} \eta}{\dot{N}}\right)^{1 / 2}\left(\frac{\lambda_{G W}}{2 \pi L_{M I G O}}\right)^{2}\left|F_{v}(f T / 4)\right|^{-1}
$$


in the shot-noise limit where $\dot{N}_{\gamma}$ is the rate photons enter LIGO.

\section{CONCLUSION}

We have shown in this paper that in terms of sensitivity, size, capability, and flexibility, the quantum methods embodied in MIGO have overwhelming advantages over the classical methods embodied in LIGO in detecting GWs. What before took many kilometers with LIGO could be done in a couple of meters, and what took millions of kilometers with LISA could be done with only hundreds of meters. Most importantly, many of the technologies needed in constructing a MIGO have already been separately demonstrated. Thus, quantum interference can become an important tool for study of fundamental problems at the intersection of quantum mechanics and general relativity [24].

\section{ACKNOWLEDGEMENTS:}

ADS and RYC were supported by a grant from the Office of Naval Research. We thank Chris McKee, John Garrison, Theodore Hänsch, Jon Magne Leinaas, Andrew P. Mackenzie,

Richard Marrus, Joseph Orenstein, William D. Phillips, and Jan Peter Toennies for many clarifying and insightful discussions.

[1] M. O. Scully, and J. P. Dowling, Phys. Rev. A48, 3186 (1993); D. Budker, D. F. Kimball, and D. P. DeMille, Atomic Physics: An Approach through Problems, (Oxford University Press, New York, 2003) p. 301.

[2] O. Stern, Naturwissensch. 17, 291 (1929).

[3] R. Collela, A. W. Overhauser, and S. A. Werner, Phys. Rev. Lett. 23, 1472 (1975).

[4] D. E. Keith, C. R. Ekstrom, Q. A. Turchette, and D. E Pritchard, Phys. Rev. Lett. 66, 2693 (1991).

[5] T. L. Gustavson, P. Bouyer, and M. A. Kasevich, Phys. Rev. Lett. 78, 2046 (1997).

[6] M. Arndt, O. Nairz, J. Voss-Andreae, C. Keller, G. Van der Zouw, and A. Zeilinger, Nature 401, 680 (1999). 
[7] Atomic and Molecular Beam Methods, Vols. 1, 2, edited by G. Scoles (Oxford University Press, New York, 1992).

[8] Private communication with J. P. Toennies.

[9] C. C. Bradley and R. Hulet in Atomic, Molecular, and Optical Physics: Atoms and Molecules, edited by F. B. Dunning, and R. G. Hulet, (Academic Press, New York, 1996) pp 129-144.

[10] D. E. Powers, et. al., J. Phys. Chem. 86, 2556 (1982).

[11] P. R. Willmott and J. R. Huber, Rev. Mod. Phys. 72, 315 (2000).

[12] A. Peters, K. Y. Chung, and S. Chu, Metrologia 38, 25 (2001).

[13] K. H. Rieder and T. Engel, Phys. Rev. Lett. 45, 824 (1980).

[14] B. Holst and W. Allison in Atomic and Molecular Beams: the State of the Art 2000, edited by R. Campargue (Springer Verlag, New York, 2001), p. 183.

[15] B. C. Barish, LIGO overview, NSF annual review of 23 October 2002, www.ligo.caltech.edu/ LIGO_web/conferences/nsf_reviews.html.

[16] N. M. Butt, J. Bashir, B. T. M. Willis, and G. Heger, Acta Cryst. A44, 396 (1988).

[17] J. H. Weare, J. Chem. Phys. 61, 2900 (1974).

[18] N. A. Robertson, Class. Quan. Grav. 17, R19 (2000).

[19] C. W. Misner, K. S. Thorne, and J. A. Wheeler, Gravitation (W. H. Freeman and Company , San Francisco, 1973), Chapters 1, 35.

[20] A. D. Speliotopoulos, Phys. Rev. D51, 1701 (1995); A. D. Speliotopoulos and R. Y. Chiao, gr-qc/0302045.

[21] J. T. Tyson and R. P. Giffard in Annual Review of Astronomy and Astrophysics, edited by G. Burbidge, D. Layzer, and J. G. Phillips, (Annual Reviews, Inc., Palo Alto, 1978) p. 521.

[22] K. S. Thorne, in 300 Years of Gravitation, edited by S. W. Hawking and W. Isreal, (Cambridge University Press, New York, 1997) p. 330.

[23] P. Fritschel in Proceedings of SPIE, Gravitational Wave Detection, Vol. 4856, edited by M. Cruise, and P. Saulson, (SPIE, Bellingham, 2003); C. Cutler and K. S. Thorne in Proceedings of the 16th International Conference on General Relativity and Gravitation, edited by N. T. Bishop and S. D. Maharaj, (World Scientific, Singapore, 2002) p. 72.

[24] R. Y. Chiao, gr-qc/0303100; R. Y. Chiao and A. D. Speliotopoulos, to appear in J. Mod. Opt., gr-qc/0312096. 